\title{
Cannabinoid Receptor 1 Gene Polymorphisms and Nonalcoholic Fatty Liver Disease in Women with Polycystic Ovary Syndrome and in Healthy Controls
}

\author{
Justyna Kuliczkowska Plaksej, ${ }^{1}$ Lukasz Laczmanski, ${ }^{1}$ \\ Andrzej Milewicz, ${ }^{1}$ A. Lenarcik-Kabza, ${ }^{1}$ Anna Trzmiel-Bira, ${ }^{1}$ \\ Urszula Zaleska-Dorobisz, ${ }^{2}$ Felicja Lwow, ${ }^{3}$ and Lidia Hirnle ${ }^{4}$ \\ ${ }^{1}$ Department of Endocrinology, Diabetology and Isotope Therapy, Wroclaw Medical University, 4 Pasteura Street, \\ 50-367 Wroclaw, Poland \\ ${ }^{2}$ Department of Radiology, Wroclaw Medical University, 68 Curie-Sklodowskiej Street, 50-369 Wroclaw, Poland \\ ${ }^{3}$ Department of Health Promotion, University School of Physical Education, 35 Paderewskiego Street, 51-612 Wroclaw, Poland \\ ${ }^{4}$ First Department of Gynaecology and Obstetrics, Wroclaw Medical University, 3 Chalubinskiego Street, 50-368 Wroclaw, Poland
}

Correspondence should be addressed to Justyna Kuliczkowska Plaksej; kuliczk@interia.pl

Received 3 February 2014; Revised 7 May 2014; Accepted 17 June 2014; Published 17 July 2014

Academic Editor: Anil K. Agarwal

Copyright (C) 2014 Justyna Kuliczkowska Plaksej et al. This is an open access article distributed under the Creative Commons Attribution License, which permits unrestricted use, distribution, and reproduction in any medium, provided the original work is properly cited.

\begin{abstract}
Context. Polycystic ovary syndrome (PCOS) is frequently associated with nonalcoholic fatty liver disease (NAFLD). The endocannabinoid system may play a crucial role in the pathogenesis of NAFLD. Polymorphism of the cannabinoid receptor 1 gene (CNR1) may be responsible for individual susceptibility to obesity and related conditions. Objective. To determine the role of genetic variants of CNR1 in the etiopathology of NAFLD in women with PCOS. Design and Setting. Our department (a tertiary referral center) conducted a cross-sectional, case-controlled study. Subjects. 173 women with PCOS (aged 20-35) and 125 healthy, ageand weight-matched controls were studied. Methods. Hepatic steatosis was assessed by ultrasound evaluation. Single nucleotide polymorphisms of CNR1 (rs806368, rs12720071, rs1049353, rs806381, rs10485170, rs6454674) were genotyped. Results. Frequency of the G allele of rs806381 $(P<0.025)$ and the GG genotype of rs10485170 $(P<0.03)$ was significantly higher in women with PCOS and NAFLD than in PCOS women without NAFLD. Frequency of the TT genotype of rs6454674 was higher in PCOS women with NAFLD (not significantly, $P=0.059$ ). In multivariate stepwise regression, allele $\mathrm{G}$ of $\mathrm{rs} 806381$ was associated with PCOS + NAFLD phenotype. Conclusion. Our preliminary results suggest the potential role of CNR1 polymorphisms in the etiology of NAFLD, especially in PCOS women.
\end{abstract}

\section{Introduction}

Polycystic ovary syndrome (PCOS) is one of the most common hormonal disorders in women in reproductive age, with a prevalence of $5-10 \%$ [1]. According to the Rotterdam criteria, its prevalence may be even as high as $18 \%$ [2]. PCOS is associated not only with alteration in sex hormones but also with metabolic disturbances such as abdominal obesity, insulin resistance (IR), atherogenic dyslipidemia, and diabetes mellitus (DM), all of them being characteristic features of metabolic syndrome (MS) [3]. Another manifestation of obesity is nonalcoholic fatty liver disease (NAFLD), with a prevalence of $70 \%$ in overweight and obese patients [4]. NAFLD prevalence is also increased in DM and dyslipidemia and, in turn, clinical features of MS are often observed in NAFLD patients [4-7]. Nowadays NAFLD is considered to be not only the hepatic manifestation of MS [8] but also an early predictor of metabolic disorders in the general population and a major cause of chronic liver disease in overweight and obese subjects [7]. Many data indicate that NAFLD and PCOS not only share features of MS but also are interconnected. There is a high proportion of NAFLD among PCOS women 
and, conversely, women with NAFLD often present many features of PCOS $[5,9,10]$. The exact etiopathology of both syndromes is still a matter of debate.

A possible mechanism that may underlie metabolic disturbances and features of PCOS and NAFLD may be dysfunction of the endocannabinoid system (EC). The EC plays a crucial role in energy homeostasis by modulation of appetite, food intake, and energy storage. Its action is transmitted by activation of two main types of receptors, 1 and 2 (CB1 and $\mathrm{CB} 2)$, located not only within the brain but also in many peripheral tissues including the gastrointestinal tract, liver, skeletal muscles, pancreas, and adipocytes [11]. Dysregulation of EC has been observed in overweight, obesity, and eating disorders and may be involved in the pathogenesis of IR, NAFLD, and MS [12-16]. In obese patients higher levels of cannabinoids in comparison to lean patients were strongly associated with visceral obesity, dyslipidemia, and IR. Blockade of CB1 inhibits food intake, promotes weight loss, inhibits adipocyte proliferation, decreases IR and waist circumference, and improves lipid profile [17-21]. CB1 has been identified in the liver and the liver has been shown to produce endocannabinoids [12, 22, 23]. During liver pathology the $\mathrm{EC}$ is activated and $\mathrm{CB} 1$ and $\mathrm{CB} 2$ are markedly upregulated, most particularly in stellate cells and vascular endothelial cells of the cirrhotic liver [24]. In animal studies, a high-fat diet increases the hepatic level of cannabinoids, density of CB1, and fatty acid synthesis, which can be reduced by CB1 blockade $[12,22,25,26]$. Activation of CB1 enhances experimental steatosis and a CB1 antagonist prevented the development of liver steatosis in rats $[12,27]$. The relationship between splanchnic EC level and liver steatosis has been recently analyzed. Results of the study of Westerbacka et al. showed that the human liver takes up cannabinoid 2-arachidonoylglycerol and produces triacylglycerols, which might reflect increased lipogenesis [28]. Exogenous phytocannabinoids also affect the severity of steatosis [29]. Moreover, data derived from clinical trials strongly suggest that selective CB1 antagonism improves IR and reduces liver fat [30].

Recently, endocannabinoid receptors and cannabinoids were also discovered within human ovaries [31,32]. CB1 and CB2 have been identified in the medulla and cortex of the ovary, in the granulosa cells of primordial, primary, secondary, and tertiary follicles, in the theca cells of secondary and tertiary follicles, and in the corpus luteum and corpus albicans. The EC is probably involved in the maturation of follicles and oocytes $[31,33]$. Cannabinoids are able to modulate the function of the hypothalamic-pituitary-gonadal axis and downregulate blood luteinizing hormone levels, by indirectly modifying gonadotropin-releasing hormone secretion in humans [34]. A direct adverse effect of cannabinoids on the ovary has been clearly documented. The role of the EC in modulation of energy balance and metabolism control could also suggest an interaction with gonadal function. Obesity and IR are associated with menstrual irregularities, chronic anovulation, and infertility. There is also a functional EC in the pancreatic islet cells and cannabinoids are released concurrently with glucose-induced insulin secretion. Some studies show a negative effect of the EC on insulin secretion while others indicate the opposite [35-38]. It is possible that the local effect of endocannabinoid signaling in the pancreas might also play a role in PCOS associated IR [32, 39]. Results of some studies have suggested that there might exist several specific, still undefined dysfunctions of the EC associated with higher prevalence of obesity and IR, which in turn are implicated in pathological conditions such as PCOS [40, 41].

We hypothesized that polymorphic variants of the $\mathrm{CB} 1$ gene (CNR1) might be associated with differences in EC activity and function and potentially contribute to individual susceptibility to obesity and related complications. The aim of this study was to investigate whether common polymorphic variants of CNR1 (rs806368, rs12720071, rs1049353, rs806381, rs10485170, and rs6454674) are associated with NAFLD frequency in PCOS women in comparison to healthy, ageand weight-matched controls.

\section{Material and Methods}

2.1. Subjects. The study group consisted of 173 women with PCOS, aged 20-35, diagnosed according to Rotterdam diagnostic criteria (2003), on the basis of two of the following features: (1) oligo- or amenorrhea, (2) clinical or biochemical hyperandrogenism, and (3) polycystic ovary in pelvic ultrasonography. Patients were excluded in case of: (1) having other causes of menstrual irregularity, (2) being pregnant, and (3) having other causes of hyperandrogenism such as hypercortisolism and 21-hydroxylase deficiency. A group of 125 healthy, age- and weight-matched controls was randomly selected from the Wroclaw city population. Women from the control group had a history of regular menstrual cycles and no evidence of hyperandrogenism. None of the women from the entire study population were on a special diet, suffered from chronic, systemic illness, smoked cigarettes, abused alcohol, used medications that influence lipid and glucose metabolism or influence liver function, or used oral contraceptive pills. Women with previously diagnosed chronic liver disorders such as viral hepatitis B and C, autoimmune hepatitis, or cirrhosis were excluded from the study. Ultrasonography of the liver and liver enzyme activity evaluation (serum aspartate and alanine aminotransferases: AST, ALT) were performed in all patients. The study group (PCOS) and control group were subdivided depending on the presence of NAFLD. All patients were informed about the aim and methods of the study and gave their written informed consent. The study protocol was approved by the Ethics Committee of Wroclaw Medical University.

2.2. Genetic Studies. Whole genomic DNA was isolated from blood leukocytes using standard methods. CNR1 genotyping (rs12720071, rs1049353, rs806368, rs806381, rs10485170, and rs6454674) was performed by two multiplex polymerase chain reactions (PCR) and minisequencing.

The first one: three fragments of the CNR1 gene (347-bp, 346-bp, and 231-bp) were amplified using multiplex PCR mix containing the specific three pairs of primers (see Table 1), 1x PCR buffer, $1.5 \mathrm{mM} \mathrm{MgCl}_{2}, 200 \mu \mathrm{M} \mathrm{dATP}, 200 \mu \mathrm{M} \mathrm{dCTP}$, $200 \mu \mathrm{M}$ dGTP, $200 \mu \mathrm{M}$ dTTP, 1x Q solution, 2 polymerase 
TABLE 1: Characteristics of study and control groups-anthropometric parameters.

\begin{tabular}{|c|c|c|c|c|c|c|}
\hline & Age & Weight (kg) & BMI $\left(\mathrm{kg} / \mathrm{m}^{2}\right)$ & Waist $(\mathrm{cm})$ & Hip (cm) & WHR \\
\hline Control - NAFLD & $27.6 \pm 6.6$ & $63.3 \pm 11.2$ & $22.7 \pm 3.6$ & $78.4 \pm 10.6$ & $100.3 \pm 14.7$ & $0.8 \pm 0.1$ \\
\hline Control + NAFLD & $27.7 \pm 5.8$ & $72.2 \pm 18.8$ & $26.4 \pm 6.3$ & $85.3 \pm 16.6$ & $105.3 \pm 12.6$ & $0.8 \pm 0.1$ \\
\hline PCOS - NAFLD & $24.1 \pm 4.4$ & $65.4 \pm 14.8$ & $24.5 \pm 8.0$ & $79.6 \pm 13.0$ & $99.2 \pm 9.4$ & $0.8 \pm 0.1$ \\
\hline PCOS + NAFLD & $25.3 \pm 5.82$ & $80.6 \pm 22.4$ & $28.7 \pm 7.4$ & $91.1 \pm 18.6$ & $106.9 \pm 12.3$ & $0.8 \pm 0.1$ \\
\hline
\end{tabular}

Data are presented as mean $\pm \mathrm{SD}$.

units (TAKARA), $200 \mathrm{ng}$ genomic DNA, and water for a total volume of $20 \mu \mathrm{L}$.

The DNA was denatured at $95^{\circ} \mathrm{C}$ for 3 minutes followed by 35 cycles of denaturation at $95^{\circ} \mathrm{C}$ for 30 seconds, annealing at $58^{\circ} \mathrm{C}$ for 30 seconds, and extension at $72^{\circ} \mathrm{C}$ for 45 seconds.

To amplify the second group of the four fragments of the CNR1 gene (205-bp, 230-bp, 280-bp and 304-bp) multiplex PCR mix was used. It was employed containing: the specific four pairs of primers (see Table 4), 1x PCR buffer, $1.5 \mathrm{mM} \mathrm{MgCl}_{2}, 200 \mu \mathrm{M}$ dATP, $200 \mu \mathrm{M}$ dCTP, $200 \mu \mathrm{M}$ dGTP, $200 \mu \mathrm{M}$ dTTP, 2 hot-start polymerase units (TAKARA), $200 \mathrm{ng}$ genomic DNA, and water for a total volume of $20 \mu \mathrm{L}$. The DNA was denatured at $95^{\circ} \mathrm{C}$ for 3 minutes followed by 35 cycles of denaturation at $95^{\circ} \mathrm{C}$ for 30 seconds, annealing at $56^{\circ} \mathrm{C}$ for 30 seconds, and extension at $72^{\circ} \mathrm{C}$ for 45 seconds.

The amplified fragments were purified from oligonucleotides and free dNTPs by SAP and ExoI treatment (Fermentas).

The minisequencing method was based on the incorporation of single fluorescence-labeled dideoxynucleotides to the $3^{\prime}$ end of the oligonucleotide that was correctly paired to the specific template DNA fragment using a SNaPshot kit (Applied Biosystems). Two SNaPshot reactions were carried out using the oligonucleotides:

(i) rs12720071: 5'-CTTGTTATGGTAGAAAAATTTCACG- $3^{\prime}$

(ii) rs1049353: 5' -TGCAGCCAGTGTTCACAGGGCCGCAGAAAGCTGCATCAAGAGCAC- $3^{\prime}$

(iii) rs806368: 5' -TTAAGATGCCACGGCAATGTAAAGAAACTCTCCCA- $3^{\prime}$

(iv) rs806381: $5^{\prime}$-TCCAACAAATGAGTGACCGTTACC $-3^{\prime}$

(v) rs10485170: 5'-ACTAGAGTTGTGCTGAGTTAATACATGAGATC-3'

(vi) rs6454674: 5'-CTTCTCCAAAATATTTCCTGGAATAAAAGAAGCAATAACT- $3^{\prime}$

designed so that it ended immediately before the polymorphic side. The SNaPshot reaction consisted of 25 cycles: denaturation at $96^{\circ} \mathrm{C}$ for 10 seconds, annealing at $50^{\circ} \mathrm{C}$ for 5 seconds, and extension at $60^{\circ} \mathrm{C}$ for 30 seconds. The product was analyzed by an ABI 3100 sequencer (Applied Biosystems). Product sizes were calculated using GeneScan 4.1 (Applied Biosystems).

2.3. Ultrasound Evaluation. Ultrasonography of the liver was performed in the morning, between 8 and 9 a.m., after an overnight fast of at least $8 \mathrm{~h}$. The mean duration of each examination was 20 minutes, which included assessment of the gall bladder, liver, and pancreas specifically and the rest of the abdomen. Diagnosis and stage of NAFLD were assessed according to the study of Saverymuttu et al. and Ma et al.: grade I (mild): increased echogenicity of liver compared with renal cortex or spleen; grade II (moderate): obscure hepatic and portal vein walls; grade III (severe): impaired visibility of the diaphragm $[42,43]$. NAFLD was also diagnosed on the basis of elevated liver enzyme activity defined as values above the upper limit of normal in our department laboratory $(>35 \mathrm{U} / \mathrm{L})$.

2.4. Statistical Analysis. The prevalence of genetic polymorphisms was analyzed in compliance with the law of HardyWeinberg. Genotype distribution was determined using the http://www.e-laboratorium.com.pl website. Statistical analysis was performed using Statistica version 10 including medical package. To verify the hypothesis of the normal distribution of numerical data the Shapiro-Wilk and KolmogorovSmirnov tests were used. To analyze the distribution of particular genotypes in both groups, Pearson's chi ${ }^{2}$ test was used. To establish the effect of CB1 receptor genotypes on clinical phenotypes in both groups we used multivariate stepwise regression analysis.

The differences were considered statistically significant at a $P$ value $\leq 0.05$.

\section{Results}

Characteristics of study and control groups are shown in Tables 1, 2, and 3 .

3.1. Frequency of NAFLD in Study Population. NAFLD was significantly more frequent in PCOS women than in the control group; it was present in 92 women (69.7\%) with PCOS versus 40 women from the control group (30.3\%) $(P<$ $0.00028, \mathrm{OR}=2.414$, and $\mathrm{RR}=1.662$ ) (Table 5).

3.2. CNR1 Polymorphisms. Genetic analysis was performed in all studied groups: 173 women with PCOS and 125 women from the control group. Frequency of the 6 assessed polymorphisms of the CNR1 gene were compared in PCOS + NAFLD versus PCOS - NAFLD and in control + NAFLD versus control - NAFLD. No statistically significant differences in CNR1 genetic variants were observed in the control group. In the PCOS group we observed significant differences in frequency of assessed polymorphisms: the G allele of rs 806381 
TABLE 2: Characteristics of study group (PCOS group).

\begin{tabular}{lccc}
\hline & PCOS + NAFLD & PCOS - NAFLD & Whole group (PCOS) \\
\hline GOT (U/L) & $24.06 \pm 11.22$ & $22.13 \pm 8.36$ & $23.24 \pm 10.13$ \\
GPT (U/L) & $27.17 \pm 18.09$ & $20.26 \pm 14.35$ & $24.25 \pm 16.92$ \\
GGTP (U/L) & $30.37 \pm 21.83$ & $21.74 \pm 13.89$ & $26.66 \pm 19.27$ \\
Bilirubin $(\mathrm{mg} / \mathrm{dL})$ & $0.677 \pm 0.364$ & $0.694 \pm 0.430$ & $0.684 \pm 0.391$ \\
Triglycerides $(\mathrm{mg} / \mathrm{dL})$ & $120.00 \pm 70.18$ & $94.60 \pm 58.52$ & $109.30 \pm 66.53$ \\
Total cholesterol (mg/dL) & $191.00 \pm 36.22$ & $185.30 \pm 37.39$ & $188.60 \pm 36.72$ \\
LDL cholesterol $(\mathrm{mg} / \mathrm{dL})$ & $113.30 \pm 32.27$ & $104.10 \pm 28.93$ & $109.50 \pm 31.17$ \\
HDL cholesterol $(\mathrm{mg} / \mathrm{dL})$ & $53.63 \pm 16.3$ & $63.68 \pm 19.63$ & $57.88 \pm 18.42$ \\
Mean glucose level $(\mathrm{mg} / \mathrm{L})$ & $113.40 \pm 23.54$ & $102.40 \pm 24.97$ & $108.70 \pm 24.7$ \\
Mean insulin level $(\mu \mathrm{IU} / \mathrm{mL})$ & $37.40 \pm 20.56$ & $50.59 \pm 37.52$ \\
Testosterone $(\mathrm{ng} / \mathrm{mL})$ & $60.46 \pm 43.89$ & $0.57 \pm 0.322$ & $0.619 \pm 0.308$ \\
SHBG (mmol/L) & $0.653 \pm 0.295$ & $46.06 \pm 29.42$ & $39.18 \pm 24.87$ \\
FAI value & $34.17 \pm 19.63$ & $6.10 \pm 5.151$ & $8.008 \pm 7.434$ \\
\hline
\end{tabular}

Data are presented as mean \pm SD.

TABLE 3: Characteristics of control group.

\begin{tabular}{|c|c|c|c|}
\hline & Control + NAFLD & Control - NAFLD & Whole group (control) \\
\hline GOT (U/L) & $20.08 \pm 7.63$ & $18.95 \pm 3.95$ & $19.51 \pm 6.06$ \\
\hline GPT (U/L) & $20.23 \pm 11.54$ & $17.19 \pm 7.47$ & $18.70 \pm 9.79$ \\
\hline GGTP (U/L) & $21.03 \pm 9.45$ & $17.76 \pm 5.59$ & $19.38 \pm 7.89$ \\
\hline Bilirubin (mg/dL) & $2.605 \pm 0.51$ & $1.325 \pm 0.78$ & $1.97 \pm 0.66$ \\
\hline Triglycerides (mg/dL) & $83.39 \pm 53.37$ & $71.63 \pm 27.21$ & $77.51 \pm 42.60$ \\
\hline Total cholesterol (mg/dL) & $181.70 \pm 37.33$ & $184.20 \pm 39.03$ & $183.00 \pm 38.09$ \\
\hline LDL cholesterol (mg/dL) & $108.60 \pm 37.18$ & $106.60 \pm 31.34$ & $107.60 \pm 34.26$ \\
\hline HDL cholesterol (mg/dL) & $66.02 \pm 21.72$ & $66.65 \pm 14.83$ & $66.33 \pm 18.52$ \\
\hline Mean glucose level (mg/L) & $101.2 \pm 19.53$ & $95.8 \pm 16.63$ & $98.4 \pm 18.25$ \\
\hline Mean insulin level $(\mu \mathrm{IU} / \mathrm{mL})$ & $40.58 \pm 35.71$ & $32.82 \pm 21.95$ & $36.67 \pm 29.72$ \\
\hline Testosterone (ng/mL) & $0.397 \pm 0.154$ & $0.368 \pm 0.163$ & $0.382 \pm 0.159$ \\
\hline SHBG $(\mathrm{mmol} / \mathrm{L})$ & $49.87 \pm 30.17$ & $58.66 \pm 25.99$ & $54.48 \pm 28.28$ \\
\hline FAI value & $3.99 \pm 3.33$ & $2.55 \pm 1.63$ & $3.23 \pm 2.67$ \\
\hline
\end{tabular}

Data are presented as mean \pm SD.

TABLE 4: Sequences of CNR1 primers.

\begin{tabular}{lll}
\hline Polymorphism & Forward primer $\left(3^{\prime}-5^{\prime}\right)$ & Reverse primer $\left(3^{\prime}-5^{\prime}\right)$ \\
\hline A3813G $($ rs12720071) & GATGAAGGCTCAGGGTGCTAGAGG & TAGTGCTGTCAGCCCCATTGTCCC \\
G1422A $($ rs1049353) & CCTGCGACACGCTTTCCGGA & CTGCCAGGGAGGCATCAGGC \\
A4895G $($ rs 806368$)$ & GAGACCACCCATATCATGCACACA & AACTCTGATCCCCAGTAGGCCTAG \\
rs806381 & CATGAGCCATGAGGTTTTCT & CATTTGAAGGCCTGTAACTT \\
rs10485170 & TTAACCAATG GTTCATCGTC & ATGTGGTTCTCAGGCATCAG \\
rs6454674 & ATGGAGCCTGTCCTTTAGGT & TATCCAGGAATGCTGCAAAA \\
\hline
\end{tabular}

TABLE 5: Frequency of NAFLD in study and control groups.

\begin{tabular}{lcc}
\hline & NAFLD & Without NAFLD \\
\hline PCOS & $92(69.70 \%)$ & $81(48.80 \%)$ \\
Control & $40(30.30 \%)$ & $85(51.20 \%)$ \\
\hline
\end{tabular}

$P<0.00028, \mathrm{OR}=2.414$, and $\mathrm{RR}=1.662$.

$(P<0.025)$ and the GG genotype of rs10485170 $(P<0.03)$ were more frequent in PCOS women with NAFLD than in
PCOS women without NAFLD. There was a higher frequency of TT genotype of rs6454674 in PCOS women with NAFLD but this association was not significant $(P=0.059)$. Frequencies of polymorphisms of CNR1 are shown in Table 6.

In stepwise regression analysis the GG genotype of rs806381 is associated with PCOS + NAFLD phenotype and increases its risk $(\mathrm{OR}=2.914 ; P=0.016)$. The GA genotype of rs806381 reduces the risk of PCOS + NAFLD phenotype by approximately $70 \%(P=0.002)$. GT genotype of rs6454674 
TABLE 6: Frequency of CNR1 polymorphisms in study and control groups.

\begin{tabular}{|c|c|c|c|c|c|c|c|c|}
\hline & \multicolumn{3}{|c|}{ A3813G (rs12720071) } & \multirow{2}{*}{$P\left(\mathrm{chi}^{2}\right)$} & \multicolumn{3}{|c|}{ A4895G (rs806368) } & \multirow{2}{*}{$P\left(\mathrm{chi}^{2}\right)$} \\
\hline & $\mathrm{A} / \mathrm{A}$ & $\mathrm{A} / \mathrm{G}$ & $\mathrm{G} / \mathrm{G}$ & & $\mathrm{T} / \mathrm{T}$ & $\mathrm{C} / \mathrm{T}$ & $\mathrm{C} / \mathrm{C}$ & \\
\hline PCOS + NAFLD & $64.41 \%(38)$ & $68.42 \%(13)$ & $50 \%(13)$ & \multirow{2}{*}{$0.35(2.04)$} & $58.82 \%(40)$ & $57.69 \%(15)$ & $87.5 \%(7)$ & \multirow{2}{*}{$0.27(2.6)$} \\
\hline PCOS - NAFLD & $35.59 \%(21)$ & $31.58 \%(6)$ & $50 \%(13)$ & & $41.18 \%(28)$ & $42.31 \%(11)$ & $12.5 \%(1)$ & \\
\hline Control + NAFLD & $48.48 \%(32)$ & $28.57 \%(4)$ & $66.67 \%(4)$ & \multirow{2}{*}{$0.24(2.89)$} & $46.3 \%(25)$ & $46.88 \%(15)$ & $100 \%(1)$ & \multirow{2}{*}{$0.57(1.14)$} \\
\hline \multirow{3}{*}{ Control - NAFLD } & $51.52 \%(34)$ & $71.43 \%(10)$ & $33.33 \%(2)$ & & $53.7 \%(29)$ & $53.13 \%(17)$ & $0.00 \%(0)$ & \\
\hline & \multicolumn{3}{|c|}{ G1422A (rs1049353) } & \multirow{2}{*}{$P\left(\mathrm{chi}^{2}\right)$} & & rs806381 & & \multirow{2}{*}{$P\left(\mathrm{chi}^{2}\right)$} \\
\hline & $\mathrm{A} / \mathrm{A}$ & G/A & $\mathrm{G} / \mathrm{G}$ & & $\mathrm{A} / \mathrm{A}$ & $\mathrm{G} / \mathrm{A}$ & $\mathrm{G} / \mathrm{G}$ & \\
\hline PCOS + NAFLD & $48 \%(12)$ & $69.7 \%(23)$ & $64.58 \%(31)$ & \multirow{2}{*}{$0.21(3.05)$} & $72 \%(18)$ & $37.5 \%(9)$ & $66.67 \%(28)$ & \multirow{2}{*}{$0.025(7.36)$} \\
\hline PCOS - NAFLD & $52 \%(13)$ & $30.3 \%(10)$ & $35.42 \%(17)$ & & $28 \%(7)$ & $62.5 \%(15)$ & $33.33 \%(14)$ & \\
\hline Control + NAFLD & $33.33 \%(4)$ & $50 \%(14)$ & $48.98 \%(24)$ & \multirow{2}{*}{$0.58(1.07)$} & $45 \%(18)$ & $50 \%(21)$ & $44.44 \%(8)$ & \multirow{2}{*}{$0.88(0.26)$} \\
\hline \multirow[t]{3}{*}{ Control - NAFLD } & $66.67 \%(8)$ & $50 \%(14)$ & $51.02 \%(25)$ & & $55 \%(22)$ & $50 \%(21)$ & $55.56 \%(10)$ & \\
\hline & & rs10485170 & & \multirow{2}{*}{$P\left(\mathrm{chi}^{2}\right)$} & & rs6454674 & & \multirow{2}{*}{$P\left(\mathrm{chi}^{2}\right)$} \\
\hline & $\mathrm{A} / \mathrm{A}$ & A/G & $\mathrm{G} / \mathrm{G}$ & & $\mathrm{T} / \mathrm{T}$ & G/T & $\mathrm{G} / \mathrm{G}$ & \\
\hline PCOS + NAFLD & $61.9 \%(52)$ & $45.45 \%(5)$ & $75 \%(6)$ & \multirow{2}{*}{$0.03(7.04)$} & $69.39 \%(34)$ & $48.89 \%(22)$ & $76.92 \%(10)$ & \multirow{2}{*}{$0.06(5.62)$} \\
\hline PCOS - NAFLD & $38.1 \%(32)$ & $54.55 \%(6)$ & $25 \%(2)$ & & $30.61 \%(15)$ & $51.11 \%(23)$ & $23.08 \%(3)$ & \\
\hline Control + NAFLD & $50 \%(41)$ & $42.86 \%(9)$ & $0 \%$ & \multirow{2}{*}{$0.56(0.34)$} & $48.98 \%(24)$ & $50 \%(20)$ & $42.86 \%(6)$ & \multirow{2}{*}{$0.89(0.22)$} \\
\hline Control - NAFLD & $50 \%(41)$ & $57.14 \%(12)$ & $0 \%$ & & $51.02 \%(25)$ & $50 \%(20)$ & $57.14 \%(8)$ & \\
\hline
\end{tabular}

PCOS + NAFLD: women with polycystic ovary syndrome and nonalcoholic fatty liver disease.

PCOS - NAFLD: women with polycystic ovary syndrome without nonalcoholic fatty liver disease.

Control + NAFLD: women from control group having nonalcoholic fatty liver disease.

Control - NAFLD: women from control group without nonalcoholic fatty liver disease.

significantly increases the risk of PCOS + NAFLD phenotype $(\mathrm{OR}=2.6 ; P=0.0130)$. There was no association between GG genotype of rs6454674 and phenotypes in the PCOS group because of the small number of carriers of GG genotype in both subgroups (only 3 cases in PCOS - NAFLD versus 10 cases in PCOS + NAFLD) (results shown in Table 7).

\section{Discussion}

In our study we observed significantly more frequent NAFLD in the group of PCOS women $(P<0.00028$, OR $=2.414$; $\mathrm{RR}=1.662)$. According to many data, there is a higher proportion of NAFLD among women with PCOS and PCOS features in women with NAFLD: $71 \%$ of NAFLD women matched the Rotterdam criteria for PCOS and $41 \%$ of PCOS women had concomitant NAFLD, whereas the incidence of NAFLD in the weight- and age-matched non-PCOS control group was only 19\% [7]. According to our study, the risk of NAFLD in the PCOS group was 2.5 times higher than that of the control group. The first evidence for the association of NAFLD and PCOS was reported in 2005 [44]. The prevalence of NAFLD in PCOS women may occur irrespectively of obesity, as reported by Gambarin-Gelwan et al. [45]. Not only components of MS but also decreased sex hormone binding globulin (SHBG) and increased free androgen index (FAI) were linked to NAFLD $[9,46]$. Therefore hyperandrogenism can be implicated with increased prevalence of NAFLD in PCOS women. Data on the association of these two disorders has yielded conflicting results. Markou et al. did not reveal any association between these two conditions in young lean women [47]. Despite this controversy, early detection of NAFLD in PCOS women is very important because early
TABLE 7: Stepwise regression analysis.

\begin{tabular}{lcccc}
\hline & $P$ & OR & CI OR 95\% & CI OR 95\% \\
\hline rs806381-GA & 0.002 & 0.308 & 0.145 & 0.654 \\
rs806381-GG & 0.016 & 2.914 & 1.220 & 6.960 \\
rs6454674-GT & 0.013 & 2.628 & 1.225 & 5.636 \\
\hline
\end{tabular}

intervention may decrease or eliminate the possibility of liver disease progression and, similarly, women with NAFLD should be routinely screened for presence of features of PCOS.

Taking into account possible involvement of the EC in NAFLD etiopathogenesis and the impact of the EC on ovaries, we investigated the link between CNR1 genotypes and NAFLD as well as PCOS. So far, one study concerning CNR1 polymorphism and NAFLD has been conducted. This study revealed that a wild variant of rs1049353 was associated with worse metabolic profile, and carriers of the A allele had a lower grade of liver fibrosis evaluated by liver biopsy. Other studies were related to the connection of common genetic variants of CNR1 with metabolic risk factors but data are contradictory. Numerous studies have revealed that polymorphisms of CNR1, rs11049353, rs12720071, rs806381, rs10485170, rs6454674, and rs2023239, were associated with features of MS such as increased BMI and waist circumference $[11,14,48]$. The $G$ allele of rs1049353 polymorphism was associated with a decreased level of adiponectin and GG homozygotes were overweight or obese [11, 48, 49]. There was also an association of rs1049353 with obesity, IR, and adipocytokines in a group of women with obesity and with fat distribution and abdominal adiposity in men [50-52]. 
An association of rs1049353 with waist circumference, waisthip ratio (WHR), and BMI was also revealed [14]. Other studies observed no association between this polymorphism and obesity, CV risk factors, or adipocytokines [51, 53, 54]. According to the study of Peeters et al. there was no significant association of this polymorphism with obesity although the $G$ allele was related to increased waist circumference and WHR [51]. In our study we found no association of rs1049353 with NAFLD in either controls or PCOS women. In the study of Benzinou et al. rs806381 was associated with obesity and BMI [55]. According to one study, it was also associated with TG level but not with BMI or WHR [56]. In our study the $G$ allele of rs806381 $(P<0.025)$ and the GG genotype of rs10485170 $(P<0.03)$ were significantly more frequent in women with PCOS and NAFLD in comparison to PCOS women without NAFLD. We also observed higher frequency of the TT genotype of rs6454674 in PCOS women with NAFLD but this association was not significant $(P=0.059)$. This might indicate the possible role of these polymorphisms in the development of NAFLD, but this association was significant only in the PCOS group. Explanation of such relationships only in PCOS women is difficult. It might result from other epigenetic (e.g., environmental) influences and different hormonal milieu which can interact with genetic factors. According to these results, we used stepwise regression to establish the effect of CB1 genotypes (rs6454674, rs806381, and rs10485170) on clinical phenotypes in both groups: PCOS + NAFLD versus PCOS - NAFLD (Table 7). We demonstrated that the GG genotype of rs806381 is associated with PCOS + NAFLD phenotype and increases its risk (OR $=2.914 ; P=0.016)$. The GA genotype of rs806381 reduces the risk of PCOS + NAFLD phenotype by approximately $70 \%$ $(P=0.002)$. In the case of rs6454674, the GT genotype significantly increases the risk of PCOS + NAFLD phenotype $(\mathrm{OR}=2.6 ; P=0.0130)$. These results might suggest that the $\mathrm{G}$ allele of rs806381 could be a risk allele for NAFLD in PCOS. Unfortunately, we did not find any association between the GG genotype of rs6454674 and phenotypes in the PCOS group, because of the small number of carriers of the GG genotype in both subgroups (only 3 cases in PCOS - NAFLD versus 10 cases in PCOS + NAFLD). These results might indicate a possible connection between CNR1 variants and etiopathology of NAFLD in PCOS women, which is complex and multifactorial. It is possible that NAFLD not only is a simple complication of MS, IR, and hyperandrogenism but also is related to genetic polymorphisms of the EC which may affect its function. We observed no significant associations between rs12720071 or rs806368 and NAFLD frequency in both study groups. Rs12720071 can influence body fat mass and fat distribution in men [57]. Carriers of the $G$ allele had a higher level of total body fat and central fat deposition [57]. Rs806368 was associated with obesity but it did not remain significant after accounting for multiple testing [58]. Results of studies are conflicting. No associations between rs806381, rs10485170, rs6454674, and rs2023239 polymorphisms and anthropometric variables were observed in a population of postmenopausal women from Poland $[59,60]$. To our knowledge, this is the first study evaluating the link between genetic variants of CNR1 and NAFLD in PCOS women.
A limitation of our study was the method used to evaluate liver steatosis. Although ultrasonography is the most widely used method for detecting liver steatosis, with an acceptable level of sensitivity, it is less sensitive than liver biopsy, which can detect $5 \%$ of fat infiltration within the liver. Another limitation was the number of genetic variants of CNR1 assessed in our study. We assessed only six common polymorphisms of CNR1 but there are also several other polymorphisms that can be associated with adverse metabolic and cardiovascular profiles, such as rs2023239, rs806378, rs806365, and rs10485179, whose relationship with obesity, type $2 \mathrm{DM}$, and IR was revealed.

In summary, our study showed significantly more frequent NAFLD in the PCOS group, which indicates that it is reasonable to carry out an ultrasound evaluation of the liver in all women with PCOS. We also observed significantly higher frequency of polymorphic variants of CNR1 ( $G$ allele of rs806381 and GG genotype of rs10485170) in women with PCOS and NAFLD in comparison with PCOS women without NAFLD. This association was found only in the PCOS group, which might result from the interaction between genetic factor and hormonal milieu. The stepwise regression analysis revealed that the $\mathrm{G}$ allele of rs806381 could be a risk allele for NAFLD in PCOS women. These results might indicate the potential impact of genetic variants of CNR1 on NAFLD etiopathology in PCOS. We did not find any relationship between the GG genotype of rs6454674 and NAFLD in PCOS possibly because of the small number of carriers of this genotype. Further studies on EC's impact on metabolic complications in PCOS with a larger number of cases are needed.

\section{Conflict of Interests}

The authors declare that there is no conflict of interests that could be perceived as prejudicing the impartiality of the research reported.

\section{Acknowledgment}

This work was supported by Grant no. NN402318839 of the Polish Ministry of Sciences.

\section{References}

[1] E. Diamanti-Kandarakis and A. G. Papavassiliou, "Molecular mechanisms of insulin resistance in polycystic ovary syndrome," Trends in Molecular Medicine, vol. 12, no. 7, pp. 324-332, 2006.

[2] F. Álvarez-Blasco, J. I. Botella-Carretero, J. L. San Millán, and H. F. Escobar-Morreale, "Prevalence and characteristics of the polycystic ovary syndrome in overweight and obese women," Archives of Internal Medicine, vol. 166, no. 19, pp. 2081-2086, 2006.

[3] H. Teede, A. Deeks, and L. Moran, "Polycystic ovary syndrome: a complex condition with psychological, reproductive and metabolic manifestations that impacts on health across the lifespan," BMC Medicine, vol. 8, article 41, 2010. 
[4] G. Vernon, A. Baranova, and Z. M. Younossi, "Systematic review: the epidemiology and natural history of non-alcoholic fatty liver disease and non-alcoholic steatohepatitis in adults," Alimentary Pharmacology and Therapeutics, vol. 34, no. 3, pp. 274-285, 2011.

[5] A. Baranova, T. P. Tran, A. Birerdinc, and Z. M. Younossi, "Systematic review: association of polycystic ovary syndrome with metabolic syndrome and non-alcoholic fatty liver disease," Alimentary Pharmacology and Therapeutics, vol. 33, no. 7, pp. 801-814, 2011.

[6] P. Angulo, "Nonalcoholic fatty liver disease," The New England Journal of Medicine, vol. 346, pp. 1221-1231, 2002.

[7] M. M. Brzozowska, G. Ostapowicz, and M. D. Weltman, "An association between non-alcoholic fatty liver disease and polycystic ovarian syndrome," Journal of Gastroenterology and Hepatology, vol. 24, no. 2, pp. 243-247, 2009.

[8] C. H. Kim and Z. M. Younossi, "Nonalcoholic fatty liver disease: a manifestation of the metabolic syndrome," Cleveland Clinic Journal of Medicine, vol. 75, no. 10, pp. 721-728, 2008.

[9] L. F. N. Zueff, W. P. Martins, C. S. Vieira, and R. A. Ferriani, "Ultrasonographic and laboratory markers of metabolic and cardiovascular disease risk in obese women with polycystic ovary syndrome," Ultrasound in Obstetrics and Gynecology, vol. 39, no. 3, pp. 341-347, 2012.

[10] N. Hossain, M. Stepanova, A. Afendy et al., "Non-alcoholic steatohepatitis (NASH) in patients with polycystic ovarian syndrome (PCOS)," Scandinavian Journal of Gastroenterology, vol. 46, no. 4, pp. 479-484, 2011.

[11] I. R. Dinu, S. G. Popa, M. MoţA et al., "The association of the rs1049353 polymorphism of the CNR1 gene with hypoadiponectinemia," Romanian Journal of Morphology and Embryology, vol. 52, no. 3, pp. 791-795, 2011.

[12] D. Osei-Hyiaman, M. DePetrillo, P. Pacher et al., "Endocannabinoid activation at hepatic CB1 receptors stimulates fatty acid synthesis and contributes to diet-induced obesity," Journal of Clinical Investigation, vol. 115, no. 5, pp. 1298-1305, 2005.

[13] K. M. Starowicz, L. Cristino, I. Matias et al., "Endocannabinoid dysregulation in the pancreas and adipose tissue of mice fed with a high-fat diet," Obesity, vol. 16, no. 3, pp. 553-565, 2008.

[14] I. R. Dinu, S. Popa, M. Bîcu, E. Moţa, and M. Moţa, “The implication of CNR1 gene's polymorphisms in the modulation of endocannabinoid system effects," Romanian Journal of Internal Medicine, vol. 47, no. 1, pp. 9-18, 2009.

[15] K. Eckardt, H. Sell, A. Taube et al., "Cannabinoid type 1 receptors in human skeletal muscle cells participate in the negative crosstalk between fat and muscle," Diabetologia, vol. 52, no. 4, pp. 664-674, 2009.

[16] A. Mallat and S. Lotersztajn, "Endocannabinoids and Liver Disease. I. Endocannabinoids and their receptors in the liver," American Journal of Physiology-Gastrointestinal and Liver Physiology, vol. 294, no. 1, pp. G9-G12, 2007.

[17] L. F. van Gaal, A. J. Scheen, A. M. Rissanen, S. Rössner, C. Hanotin, and $\mathrm{O}$. Ziegler, "Long-term effect of $\mathrm{CB}_{1}$ blockade with rimonabant on cardiometabolic risk factors: two year results from the RIO-Europe study," European Heart Journal, vol. 29, no. 14, pp. 1761-1771, 2008.

[18] J. P. Després, A. Golay, and L. Sjöström, "Effects of rimonabant on metabolic risk factors in overweight patients with dyslipidemia," The New England Journal of Medicine, vol. 353, no. 20, pp. 2121-2134, 2005.

[19] F. X. Pi-Sunyer, L. J. Aronne, H. M. Heshmati, J. Devin, and J. Rosenstock, "Effect of rimonabant, a cannabinoid-1 receptor blocker, on weight and cardiometabolic risk factors in overweight or obese patients: RIO-North America: a randomized controlled trial," Journal of the American Medical Association, vol. 295, no. 7, pp. 761-775, 2006.

[20] A. J. Scheen, N. Finer, P. Hollander, M. D. Jensen, and L. F. van Gaal, "Efficacy and tolerability of rimonabant in overweight or obese patients with type 2 diabetes: a randomised controlled study," The Lancet, vol. 368, no. 9548, pp. 1660-1672, 2006.

[21] J. Rosenstock, P. Hollander, S. Chevalier, and A. Iranmanesh, "SERENADE: the study evaluating rimonabant efficacy in drugnaive diabetic patients: effects of monotherapy with rimonabant, the first selective CB 1 receptor antagonist, on glycemic control, body weight, and lipid profile in drug-naive type 2 diabetes," Diabetes Care, vol. 31, no. 11, pp. 2169-2176, 2008.

[22] W. Jeong, D. Osei-Hyiaman, O. Park et al., "Paracrine activation of hepatic cbl receptors by stellate cell-derived endocannabinoids mediates alcoholic fatty liver," Cell Metabolism, vol. 7, no. 3, pp. 227-235, 2008.

[23] F. Teixeira-Clerc, B. Julien, P. Grenard et al., "CB1 cannabinoid receptor antagonism: a new strategy for the treatment of liver fibrosis," Nature Medicine, vol. 12, no. 6, pp. 671-676, 2006.

[24] W. I. Jeong, D. Osei-Hyiaman, O. Park et al., "Paracrine activation of hepatic $\mathrm{CB1}$ receptors by stellate cell-derived endocannabinoids mediates alcoholic fatty liver," Cell Metabolism, vol. 7, no. 3, pp. 227-235, 2008.

[25] P. Gazzerro, M. G. Caruso, M. Notarnicola et al., "Association between cannabinoid type-1 receptor polymorphism and body mass index in a southern Italian population," International Journal of Obesity, vol. 31, no. 6, pp. 908-912, 2007.

[26] D. Osei-Hyiaman, J. Liu, L. Zhou et al., "Hepatic CB1 receptor is required for development of diet-induced steatosis, dyslipidemia, and insulin and leptin resistance in mice," Journal of Clinical Investigation, vol. 118, no. 9, pp. 3160-3169, 2008.

[27] M. Gary-Bobo, G. Elachouri, J. F. Gallas et al., "Rimonabant reduces obesity-associated hepatic steatosis and features of metabolic syndrome in obese zucker fa/fa rats," Hepatology, vol. 46, no. 1, pp. 122-129, 2007.

[28] J. Westerbacka, A. Kotronen, B. A. Fielding et al., "Splanchnic balance of free fatty acids, endocannabinoids, and lipids in subjects with nonalcoholic fatty liver disease," Gastroenterology, vol. 139, no. 6, pp. 1961-1971, 2010.

[29] C. Hézode, E. S. Zafrani, F. Roudot-Thoraval et al., "Daily cannabis Use: a novel risk factor of steatosis severity in patients with chronic hepatitis C," Gastroenterology, vol. 134, no. 2, pp. 432439, 2008.

[30] K. A. Alswat, "The role of endocannabinoids system in fatty liver disease and therapeutic potentials," Saudi Journal of Gastroenterology, vol. 19, no. 4, pp. 144-151, 2013.

[31] M. R. El-Talatini, A. H. Taylor, J. C. Elson, L. Brown, A. C. Davidson, and J. C. Konje, "Localisation and function of the endocannabinoid system in the human ovary," PLoS ONE, vol. 4, no. 2, Article ID e4579, 2009.

[32] A. M. Di Blasio, M. Vignali, and D. Gentilini, "The endocannabinoid pathway and the female reproductive organs," Journal of Molecular Endocrinology, vol. 50, no. 1, pp. R1-R9, 2012.

[33] M. R. El-Talatini, J. C. Elson, A. H. Taylor, and J. C. Konje, "The endocannabinoid, anandamide, is involved in human folliculogenesis and oocyte maturation during IVF treatment," in Proceedings of the 2nd SGI International Summit in Reproductive Medicine-From Embryo and Endometrium to Implantation, p. 59, Valencia, Spain, 2007. 
[34] L. L. Murphy, R. W. Steger, M. S. Smith, and A. Bartke, "Effects of delta-9-tetrahydrocannabinol, cannabinol and cannabidiol, alone and in combinations, on luteinizing hormone and prolactin release and on hypothalamic neurotransmitters in the male rat," Neuroendocrinology, vol. 52, no. 4, pp. 316-321, 1990.

[35] I. Matias, M. Gonthier, P. Orlando et al., "Regulation, function, and dysregulation of endocannabinoids in models of adipose and $\beta$-pancreatic cells and in obesity and hyperglycemia," Journal of Clinical Endocrinology and Metabolism, vol. 91, no. 8, pp. 3171-3180, 2006.

[36] F. J. Bermúdez-Silva, J. Suárez, E. Baixeras et al., "Presence of functional cannabinoid receptors in human endocrine pancreas," Diabetologia, vol. 51, no. 3, pp. 476-487, 2008.

[37] F. J. Bermúdez-Silva, J. Suárez Pérez, A. Nadal, and F. Rodríguez de Fonseca, "The role of the pancreatic endocannabinoid system in glucose metabolism," Best Practice \& Research Clinical Endocrinology \& Metabolism, vol. 23, pp. 87-102, 2009.

[38] W. Kim, M. E. Doyle, Z. Liu et al., "Cannabinoids inhibit insulin receptor signaling in pancreatic $\beta$-cells," Diabetes, vol. 60 , no. 4 , pp. 1198-1209, 2011.

[39] R. Pasquali, L. Patton, and A. Gambineri, "Obesity and infertility," Current Opinion in Endocrinology, Diabetes and Obesity, vol. 14, no. 6, pp. 482-487, 2007.

[40] R. Pasquali, A. Gambineri, and U. Pagotto, "The impact of obesity on reproduction in women with polycystic ovary syndrome," BJOG: An International Journal of Obstetrics and Gynaecology, vol. 113, no. 10, pp. 1148-1159, 2006.

[41] N. Battista, N. Pasquariello, M. di Tommaso, and M. Maccarrone, "Interplay between endocannabinoids, steroids and cytokines in the control of human reproduction," Journal of Neuroendocrinology, vol. 20, no. 1, pp. 82-89, 2008.

[42] S. H. Saverymuttu, A. E. A. Joseph, and J. D. Maxwell, "Ultrasound scanning in the detection of hepatic fibrosis and steatosis," British Medical Journal, vol. 292, no. 6512, pp. 13-15, 1986.

[43] X. Ma, N. S. Holalkere, R. A. Kambadakone, M. Mino-Kenudson, P. F. Hahn, and D. V. Sahani, "Imaging-based quantification of hepatic fat: methods and clinical applications," Radiographics, vol. 29, no. 5, pp. 1253-1277, 2009.

[44] A. J. Brown, D. A. Tendler, R. G. McMurray, and T. L. Setji, "Polycystic ovary syndrome and severe nonalcoholic steatohepatitis: beneficial effect of modest weight loss and exercise on liver biopsy findings," Endocrine Practice, vol. 11, no. 5, pp. 319324, 2005.

[45] M. Gambarin-Gelwan, S. V. Kinkhabwala, T. D. Schiano, C. Bodian, H. Yeh, and W. Futterweit, "Prevalence of nonalcoholic fatty liver disease in women with polycystic ovary syndrome," Clinical Gastroenterology and Hepatology, vol. 5, no. 4, pp. 496501, 2007.

[46] H. Jones, V. S. Sprung, C. J. A. Pugh et al., "Polycystic ovary syndrome with hyperandrogenism is characterized by an increased risk of hepatic steatosis compared to nonhyperandrogenic PCOS phenotypes and healthy controls, independent of obesity and insulin resistance," Journal of Clinical Endocrinology and Metabolism, vol. 97, no. 10, pp. 3709-3716, 2012.

[47] A. Markou, I. I. Androulakis, C. Mourmouris et al., "Hepatic steatosis in young lean insulin resistant women with polycystic ovary syndrome," Fertility and Sterility, vol. 93, no. 4, pp. 12201226, 2010.

[48] D. A. de Luis, M. González Sagrado, R. Aller, O. Izaola, R. Conde, and E. Romero, "G1359A polymorphism of the cannabinoid receptor gene (CNR1) and insulin resistance in patients with diabetes mellitus type 2," Nutricion Hospitalaria, vol. 25, no. 1, pp. 34-38, 2010.

[49] D. A. de Luis, M. González Sagrado, R. Aller, O. Izaola, and R. Conde, "Influence of G1359A polymorphism of the cannabinoid receptor gene on anthropometric parameters and insulin resistance in women with obesity," Metabolism: Clinical and Experimental, vol. 60, no. 2, pp. 272-276, 2011.

[50] A. Peeters, S. Beckers, I. Mertens, W. van Hul, and L. van Gaal, "The G1422A variant of the cannabinoid receptor gene (CNR1) is associated with abdominal adiposity in obese men," Endocrine, vol. 31, no. 2, pp. 138-141, 2007.

[51] M. Frost, T. L. Nielsen, K. Wraae et al., "Polymorphisms in the endocannabinoid receptor 1 in relation to fat mass distribution," European Journal of Endocrinology, vol. 163, no. 3, pp. 407-412, 2010.

[52] W. Lieb, A. K. Manning, J. C. Florez et al., "Variants in the CNR1 and the FAAH genes and adiposity traits in the community," Obesity, vol. 17, no. 4, pp. 755-760, 2009.

[53] T. D. Müller, K. Reichwald, A. Wermter et al., "No evidence for an involvement of variants in the cannabinoid receptor gene (CNR1) in obesity in German children and adolescents," Molecular Genetics and Metabolism, vol. 90, no. 4, pp. 429-434, 2007.

[54] E. Völgyi, F. A. Tylavsky, A. Lyytikäinen, H. Suominen, M. Alén, and S. Cheng, "Assessing body composition with DXA and bioimpedance: effects of obesity, physical activity, and age," Obesity, vol. 16, no. 3, pp. 700-705, 2008.

[55] M. Benzinou, J. Chèvre, K. J. Ward et al., "Endocannabinoid receptor 1 gene variations increase risk for obesity and modulate body mass index in European populations," Human Molecular Genetics, vol. 17, no. 13, pp. 1916-1921, 2008.

[56] M. Zhuang, Y. Yang, F. Cao et al., "Associations of variants of CNR1 with obesity and obesity-related traits in Chinese women," Gene, vol. 495, no. 2, pp. 194-198, 2012.

[57] P. Russo, P. Strazzullo, F. P. Cappuccio et al., "Genetic variations at the endocannabinoid type 1 receptor gene (CNR1) are associated with obesity phenotypes in men," Journal of Clinical Endocrinology and Metabolism, vol. 92, no. 6, pp. 2382-2386, 2007.

[58] D. Schleinitz, S. Carmienke, Y. Böttcher et al., "Role of genetic variation in the cannabinoid type 1 receptor gene (CNR1) in the pathophysiology of human obesity," Pharmacogenomics, vol. 11, no. 5, pp. 693-702, 2010.

[59] A. Milewicz, U. Tworowska-Bardzińska, D. Jedrzejuk et al., "Are endocannabinoid type 1 receptor gene (CNR1) polymorphisms associated with obesity and metabolic syndrome in postmenopausal Polish women?" International Journal of Obesity, vol. 35, no. 3, pp. 373-377, 2011.

[60] Ł. Łaczmański, A. Milewicz, K. Dunajska, D. Jedrzejczuk, M. Pawlak, and F. Lwow, "Endocannabinoid type 1 receptor gene (CNR1) polymorphisms (rs806381, rs10485170, rs6454674, rs2023239) and cardiovascular risk factors in postmenopausal women," Gynecological Endocrinology, vol. 27, no. 12, pp. 10231027, 2011. 


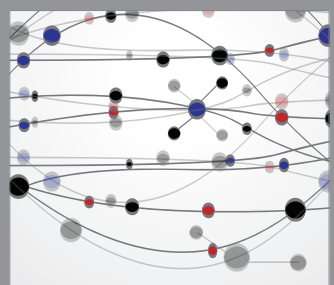

The Scientific World Journal
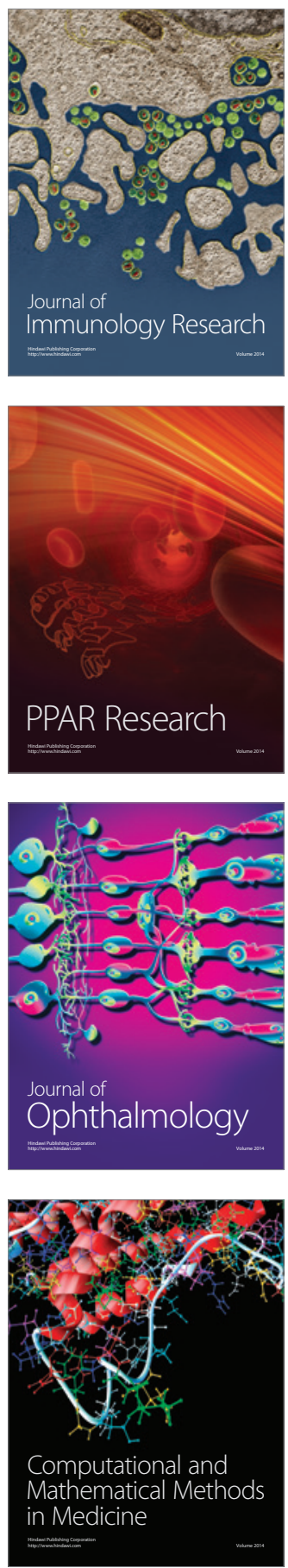

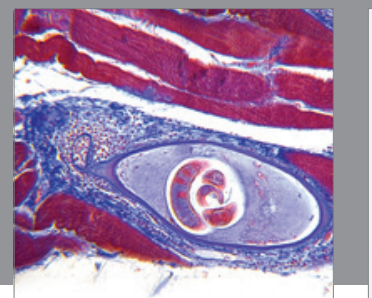

Gastroenterology

Research and Practice
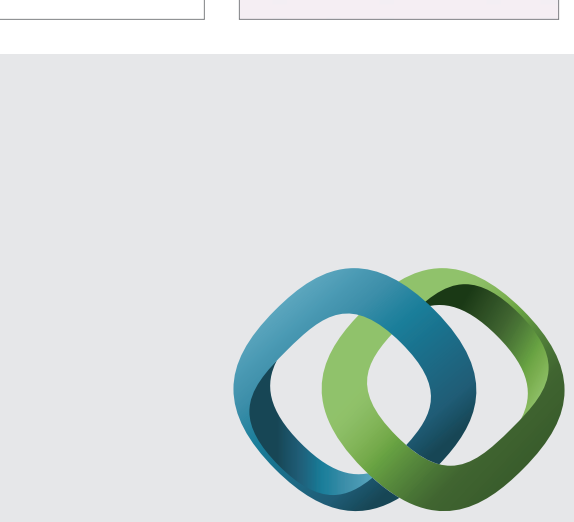

\section{Hindawi}

Submit your manuscripts at

http://www.hindawi.com
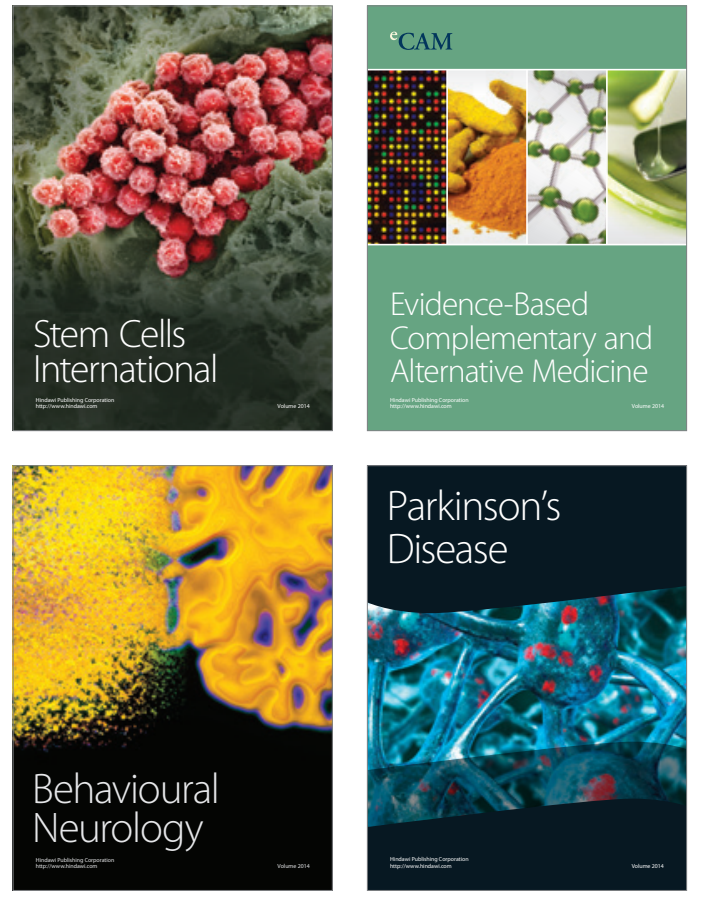
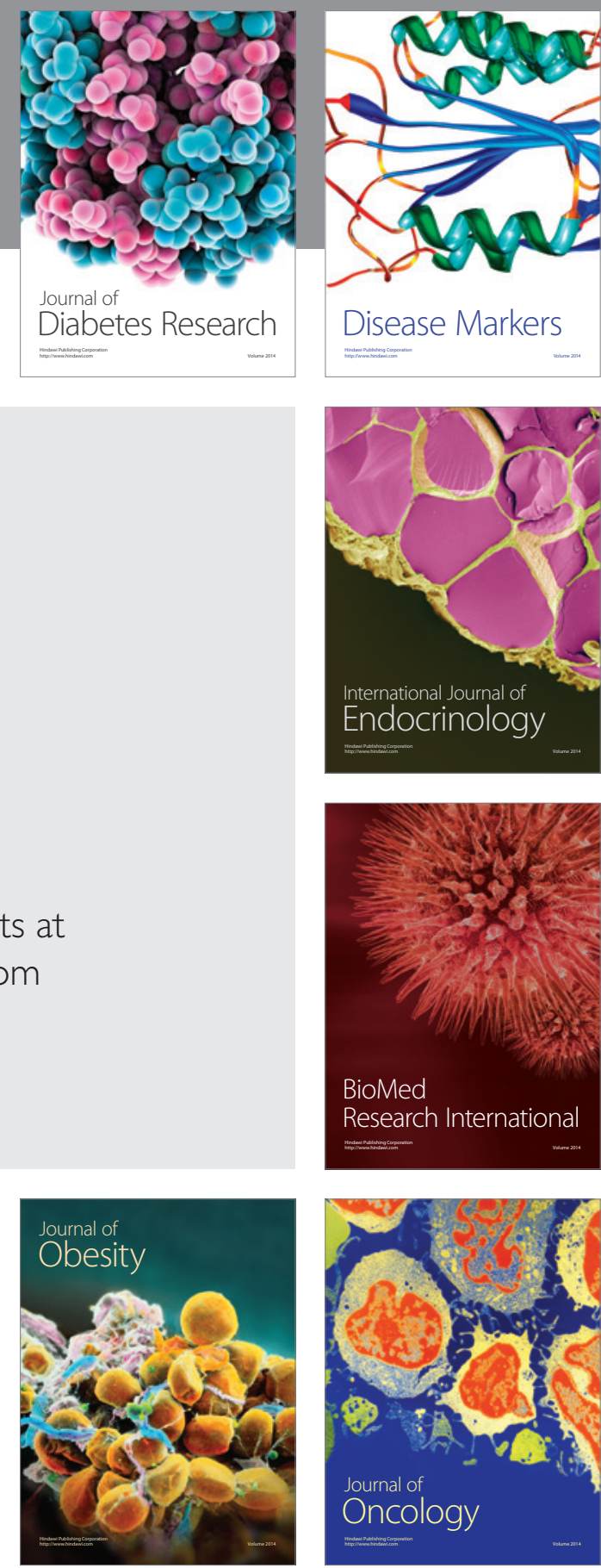

Disease Markers
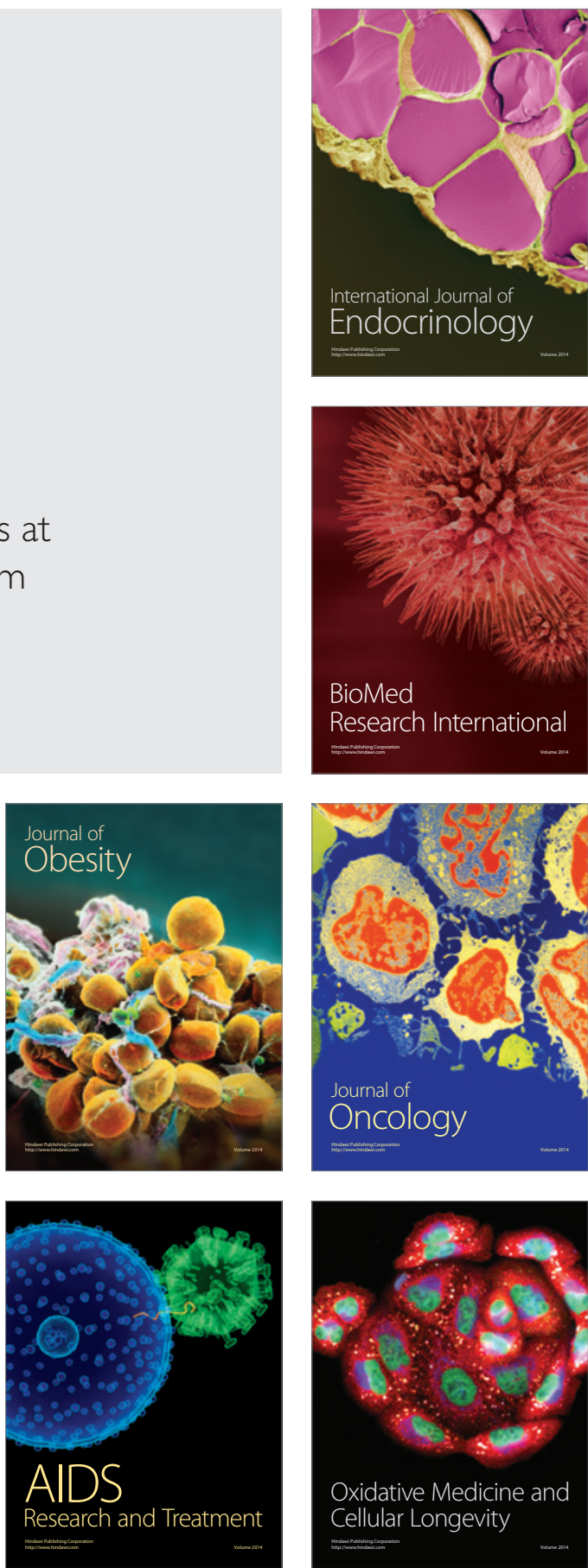